Ibn Al-Haitham Jour. for Pure \& Appl. Sci. 33 (4) 2020

Ibn Al Haitham J ournal for Pure and Applied Science

J ournal homepage: http://jih.uobaghdad.edu.iq/index.php/j/index

\title{
Fuzzy Semi Pre Homeomorphism in Fuzzy Topological Spaces
}

\author{
Saleem Y. Majeed \\ Department of Mathematics, College of Education, University of Garmian \\ Saleem.yaseen@garmian.edu.krd
}

Article history: Received,2019, Accepted,2020, Published in October 2020

Doi: 10.30526/33.4.2511

\begin{abstract}
The aim of this paper is to introduce and study new class of fuzzy function called fuzzy semi pre homeomorphism in a fuzzy topological space by utilizing fuzzy semi pre-open sets. Therefore, some of their characterization has been proved; In addition to that we define, study and develop corresponding to new class of fuzzy semi pre homeomorphism in fuzzy topological spaces using this new class of functions.
\end{abstract}

Keyword: Fuzzy topological spaces, fuzzy semi pre-open sets, fuzzy semi pre homeomorphism function and fuzzy semi pre* homeomorphism function.

\section{Introduction}

Zadeh in [1] introduced the fundamental concept of fuzzy sets and fuzzy set operations in his standard paper. Thereafter, some researchers have applied some different basic notions from general topology by utilizing the fundamental idea of fuzzy sets and was subsequently expanded by developing important theories of fuzzy topological spaces. The notion of family in fuzzy sets naturally plays a very significant role in the study of the recent concept of fuzzy topology introduced by Chang [2]. While, Ming, P. \& Ming, L. [3] introduced the concept of extensions principle of functions in fuzzy setting.

The idea of semi pre-open set in classical topology was introduced by Andrijevic, D. [4]. The conception of fuzzy semi pre-open set and fuzzy semi pre-open continuity in fuzzy topological spaces was introduced by Thakur, S. S. and Singh, S. [5] and investigated some of their properties.

This paper is to introduce and investigate some new types of fuzzy semi pre continuous functions and fuzzy semi pre homeomorphism functions via fuzzy semi pre-open sets. Also, the relationships between these functions and other types are discussed. Several properties of 
these new notions are investigated and the connections between them are studied. Moreover, some results in this topic with some properties and corollaries are developed.

\section{Preliminaries}

Throughout this paper $X$ denotes anon empty set and $I$ denote the interval $[0,1]$. A fuzzy set in $X$ is a function $\mu$ from $X$ in to $I$. The value $\mu_{\tilde{A}}(x)$ represents the degree of membership of $x \in X$ in the fuzzy set A. The family of all fuzzy sets on a crisp set $X$ will be denoted by $I^{X}$. A family $\tilde{\tau}$ of fuzzy sets in $X$ is called a fuzzy topology for $X$ iff (1) $\tilde{0}, \tilde{1} \in \tilde{\tau}$ (2) for all $\tilde{A}, \quad \tilde{B} \in \tilde{\tau}$ then $\operatorname{Min}\left\{\mu_{\tilde{A}}(x), \mu_{\tilde{B}}(x)\right\} \in \tilde{\tau}$ (3) if $\tilde{A}_{i} \in \tilde{\tau}$ for each $i \in I$, then $\operatorname{Sup}_{i \in I}\left\{\mu_{\tilde{A}_{i}}(x)\right\} \in \tilde{\tau}$. Moreover, the pair $(X, \tilde{\tau})$ is called a fuzzy topological space (abbreviated as f.t.s) and every member of $\tilde{\tau}$ is called a fuzzy open set [2].

A fuzzy set in a crisp set $X$ is called a fuzzy point if it takes the grade of membership (0) for all $x \in X-\{1\}$, say, $p \in X$. If its grade of membership at $x$ is $\alpha(\tilde{0}<\alpha \leq \tilde{1})$, we denote this fuzzy point by $p_{\alpha}$, where the point $p$ is called its support [6]. For any fuzzy $p_{\varepsilon}$ and any fuzzy set $\tilde{A}$, we write $p_{\varepsilon} \in \tilde{A}$ iff $\varepsilon \leq \mu_{\tilde{A}}(x)$.

\section{Definition 2.1 [7]:}

Let $f:(X, \tilde{\tau}) \longrightarrow(Y, \tilde{v})$, let $\tilde{B}$ be a fuzzy set in Y with membership function $\mu_{\tilde{B}}(y)$. Then the inverse of $\tilde{B}$ written as $f^{-1}(\tilde{B})$ is a fuzzy set in X whose membership function is defined by $\mu_{f^{-1}(\tilde{B})}(x)=\mu_{\tilde{B}}(f(x))$ for all $x \in X$.

Conversely, let $\tilde{A}$ be a fuzzy set in $\mathrm{X}$ with membership function $\mu_{\tilde{A}}(x)$. The image of $\tilde{A}$ written as $f(\tilde{A})$ is a fuzzy set in Y whose membership function is given by

$$
\mu_{f(\tilde{A})}(Y)=\left\{\begin{array}{cc}
\operatorname{Sup}_{x \in f^{-1}(y)}\left\{\mu_{\tilde{A}}(x)\right\}, & \text { if } \mu_{f^{-1}(y)}(x) \text { is not empty } \\
0, & \text { otherwise }
\end{array}\right.
$$

For each y belong to $\mathrm{Y}$, where $\mu_{f^{-1}(y)}(x)=\mu_{f(x)}(Y)=\mu_{y}(Y)$.

\section{Definition 2.2:}

Let $f:(X, \tilde{\tau}) \longrightarrow(Y, \tilde{v})$, then $f$ is called:

(1) "A fuzzy continuous function" if the $\mu_{f^{-1}(y)}(x)$ is a fuzzy open set in $\mathrm{X}$ for each $y \in \tilde{v}$ and denoted (f.co.f.). [8]

(2) "A fuzzy open function" if the $\mu_{f(x)}(Y)$ is a fuzzy open set in $\mathrm{Y}$, for each $x \in \tilde{\tau}$ and denoted (f.o.f.). [9]

(3) "A fuzzy closed function" if the $\mu_{f(x)}(Y)$ is a fuzzy closed set in Y, for each $x^{c} \in \tilde{\tau}$ and denoted (f.cl.f.). [10]

\section{Definition 2.3 [2]:}

Let $f:(X, \tilde{\tau}) \longrightarrow(Y, \tilde{v})$ bijection fuzzy function, $f$ is called a fuzzy homeomorphism if $f$ and $f^{-1}$ are f.co.f. (abbreviated as f.h.f.) 


\section{Theorem 2.4 [6]:}

Let $f:(X, \tilde{\tau}) \longrightarrow(Y, \tilde{v})$, Then all the below are true:

(1) For any fuzzy set $\tilde{B}$ in $\mathrm{Y}, \mu_{f^{-1}\left(1-\mu_{\widetilde{B}(y)}\right)}(x)=1-\mu_{f^{-1}(\tilde{B})}(x)$.

(2) For any fuzzy set $\tilde{A}$ in $\mathrm{X}, 1-\mu_{f(\tilde{A})}(Y)=\mu_{f\left(1-\mu_{\widetilde{A}(x)}\right)}(Y)$.

(3) For any fuzzy set $\tilde{A}$ in $\mathrm{X}, \mu_{\tilde{A}}(x) \leq \mu_{f^{-1}(f(\tilde{A}))}(x)$ and $\mu_{\tilde{A}}(x)=\mu_{f^{-1}(f(\tilde{A}))}(x)$ if $f$ is one-to-one.

(4) For any fuzzy set $\tilde{B}$ in Y, $\mu_{f\left(f^{-1}(\tilde{B})\right)}(y) \leq \mu_{\tilde{B}}(y)$ and $\mu_{f\left(f^{-1}(\tilde{B})\right)}(y)=\mu_{\tilde{B}}(y)$ if $f$ is onto.

\section{Fuzzy Semi Pre Open Sets}

This section is devoted to definitions, and some theorems of fuzzy semi pre-open set and fuzzy semi pre closed set. Moreover, we study in this section some remarks and relationship between fuzzy semi pre-open (semi pre closed) set and fuzzy open (closed) set. Also some of their properties which we need them in our study are discussed.

\section{Definition 3.1 [11]:}

Let $(X, \tilde{\tau})$ be a f.t.s, a fuzzy set $\tilde{A}$ in $\mathrm{X}$ is called:

(1) Fuzzy semi pre-open set if $\mu_{\tilde{A}}(x) \leq \mu_{c l(\operatorname{int}(\operatorname{cl}(\tilde{A})))}(x)$.

(2) Fuzzy semi pre closed set if $\mu_{\text {int }(\operatorname{cl}(\operatorname{int}(\tilde{A})))}(x) \leq \mu_{\tilde{A}}(x)$.

\section{Notation 3.2:}

In f.t.s $(X, \tilde{\tau})$, we denote:

(1) The family of all fuzzy semi pre-open sets of X by F. S. P. O. (X).

(2) The family of all fuzzy semi pre closed sets of X by F. S. P. C. (X).

\section{Theorem 3.3 [5]:}

Let $\tilde{\rho}$ in f.t.s $(X, \tilde{\tau}), \mu_{\widetilde{\rho}}(x) \in$ F.S.P.C(X) if and only if $1-\mu_{\widetilde{\rho}}(x) \in$ F.S.P.O.(X).

\section{Remark 3.4:}

Every fuzzy open (resp. closed) set is fuzzy semi pre-open (resp. semi pre closed) set. But the converse is not true in general.

\section{Example 3.5:}

Let $X=\{\alpha, \beta\}$ and let $\tilde{A}, \tilde{B}$ are fuzzy sets of $X$ defined as follows:

$$
\begin{array}{llll}
\mu_{\tilde{A}}(\alpha)=0.4 & \mu_{\tilde{A}}(\beta)=0.4 & \mu_{\tilde{B}}(\alpha)=0.7 & \mu_{\tilde{B}}(\beta)=0.7 \\
\mu_{\tilde{C}}(\alpha)=0.8 & \mu_{\tilde{C}}(\beta)=0.6 & \mu_{\tilde{E}}(\alpha)=0.3 & \mu_{\tilde{E}}(\beta)=0.7
\end{array}
$$

Let $\tilde{\tau}=\{\tilde{0}, \tilde{1}, \tilde{A}, \tilde{B}\}$ be a f.t.s. on $X$, it's clear that:

(1) The fuzzy set $\tilde{C}$ is fuzzy semi pre-open set in X but it is not a fuzzy open set.

(2) The fuzzy set $\tilde{E}$ is fuzzy semi pre closed set but it is not a fuzzy closed set.

\section{Theorem 3.6 [5]:}

Let $(X, \tilde{\tau})$ be a f.t.s, then: 
(1) Let $\tilde{A}_{i} \in$ F.S. P. O. (X) for each $i \in I$ then $\operatorname{Sup}_{i \in I}\left\{\mu_{\tilde{A}_{i}}(x)\right\} \in$ F. S. P. O. (X).

(2) Let $\tilde{B}_{i} \in$ F.S.P.C. (X) for each $i \in I$ then $\operatorname{Inf}_{i \in I}\left\{\mu_{\tilde{B}_{i}}(x)\right\} \in$ F.S. P. C. (X).

\section{Remark 3.7 [5]:}

(1) Let $\tilde{\delta}$ and $\tilde{\vartheta}$ be two fuzzy semi pre-open sets then $\operatorname{Min}\left\{\mu_{\widetilde{\delta}}(x), \mu_{\widetilde{\vartheta}}(x)\right\}$ need not to be fuzzy semi pre open set.

(2) Let $\tilde{\rho}$ and $\tilde{\sigma}$ be two fuzzy semi pre closed sets then $\operatorname{Max}\left\{\mu_{\widetilde{\rho}}(x), \mu_{\widetilde{\sigma}}(x)\right\}$ need not to be fuzzy semi pre closed set.

\section{Example 3.8:}

Let $X=\{\alpha, \beta\}$ and let $\tilde{A}, \tilde{B}$ are fuzzy sets of $X$ defined as follows:

$$
\begin{array}{llll}
\mu_{\tilde{A}}(\alpha)=0.6 & \mu_{\tilde{A}}(\beta)=0.5 & \mu_{\tilde{B}}(\alpha)=0.7 & \mu_{\tilde{B}}(\beta)=0.7 \\
\mu_{\tilde{C}}(\alpha)=0.9 & \mu_{\tilde{C}}(\beta)=0.4 & \mu_{\widetilde{D}}(\alpha)=0.4 & \mu_{\widetilde{D}}(\beta)=0.8 \\
\mu_{\tilde{E}}(\alpha)=0.4 & \mu_{\tilde{E}}(\beta)=0.8 & \mu_{\tilde{F}}(\alpha)=0.7 & \mu_{\tilde{F}}(\beta)=0.4
\end{array}
$$

Let $\tilde{\tau}=\{\tilde{0}, \tilde{1}, \tilde{A}, \tilde{B}\}$ be a f.t.s. on $X$, it's clear that:

(1) The fuzzy set $\tilde{C}$ and $\widetilde{D}$ are fuzzy semi pre-open sets in X, but $\operatorname{Min}\left\{\mu_{\tilde{C}}(x), \mu_{\widetilde{D}}(x)\right\}$ is not a fuzzy semi pre-open set.

(2) The fuzzy set $\tilde{E}$ and $\tilde{F}$ are fuzzy semi pre closed sets in X, but $\operatorname{Max}\left\{\mu_{\tilde{E}}(x), \mu_{\tilde{F}}(x)\right\}$ is not a fuzzy semi pre closed set.

\section{Definition 3.9:}

Let $f:(X, \tilde{\tau}) \longrightarrow(Y, \tilde{v})$, then $f$ is called:

(1)"A fuzzy semi pre-open function" if the $\mu_{f(x)}(Y) \in$ F.S.P.O. $(Y)$, for each $x \in \tilde{\tau}$ and denoted(f.s.p.o.f.). [5]

(2) "A fuzzy semi pre closed function" if the $\mu_{f(x)}(Y) \in$ F.S.P.C. $(Y)$, for each $x^{c} \in \tilde{\tau}$ and denoted(f.s.p.cl.f.). [5]

(3)"A fuzzy semi pre*- open function" if the $\mu_{f(x)}(Y) \in$ F.S.P.O.(Y), for each $x \in$ F.S.P.O. (X) and denoted(f.s.p. ${ }^{*}$.o.f.).

(4) "A fuzzy semi pre* closed function" if the $\mu_{f(x)}(Y) \in$ F.S.P.C. $(Y)$, for each $x \in$ F.S.P.C. (X) and denoted(f.s.p*..cl.f.).

\section{Definition 3.10:}

Let $\mathrm{f}:(\mathrm{X}, \tilde{\mathrm{\tau}}) \longrightarrow(\mathrm{Y}, \tilde{\mathrm{v}})$, then $\mathrm{f}$ is called:

(1) "A fuzzy semi pre continuous function" if the $\mu_{\mathrm{f}^{-1}(\mathrm{y})}(\mathrm{x}) \in$ F.S.P. O. (X) for each $y \in \tilde{v}$ and denoted (f. s. p. co.f.). [11]

(2) "A fuzzy semi pre irresolute function" if the $\mu_{\mathrm{f}^{-1}(\mathrm{y})}(\mathrm{x}) \in$ F.S.P.O. (X) for each y $\in$ F. S. P. O. (Y) and denoted (f. s. p.i.f.). [11]

(3) "A fuzzy semi pre* continuous function" if the $\mu_{\mathrm{f}^{-1}(\mathrm{y})}(\mathrm{x})$ is fuzzy open set in $\mathrm{X}$ for each $y \in$ F. S. P. O. (Y) and denoted (f. s. p*. co.f.). 


\section{Proposition 3.11:}

(1) Every f.s. p*.co.f. is f.co.f.

(2) Every f.co.f. is f.s.p.co.f.

(3) Every f.s.p*.co.f. is f.s.p.co.f.

(4) Every f.s.p ${ }^{*}$.co.f. is f.s.p.i.f.

(5) Every f.s.p.i.f. is f.s.p.co.f.

\section{Remark 3.12:}

The converse of proposition (3.11) is not true in general.

\section{Example 3.13:}

Let $X=\{\alpha, \beta\}, Y=\{\rho, \sigma\}$ and let $\tilde{\delta}, \tilde{\vartheta}, \tilde{\mathcal{G}}$ be fuzzy sets defined as follows:

$$
\begin{array}{ll}
\mu_{\widetilde{\delta}}(\alpha)=0.6 & \mu_{\widetilde{\delta}}(\beta)=0.5 \\
\mu_{\widetilde{\vartheta}}(\rho)=0.7 & \mu_{\widetilde{\vartheta}}(\sigma)=0.7 \\
\mu_{\tilde{\mathcal{G}}}(\rho)=0.9 & \mu_{\tilde{\mathcal{G}}}(\sigma)=0.7
\end{array}
$$

Let $\tilde{\tau}=\{\tilde{0}, \tilde{1}, \tilde{\delta}, \tilde{\vartheta}\}, \quad \tilde{v}=\{\tilde{0}, \tilde{1}, \tilde{\vartheta}\} \quad$ so, $(X, \tilde{\tau})$ and $(Y, \tilde{v})$ are f.t.s. Then the function $f:(X, \tilde{\tau}) \longrightarrow(Y, \tilde{v})$ defined by $f(\alpha)=\rho, f(\beta)=\sigma$ is $f . c o . f$ and f.s.i.f but not f.s. $p^{*} . \cos f$.

\section{Example 3.14:}

Let $X=\{\alpha, \beta, \lambda\}, Y=\{\rho, \sigma, \omega\}$ and let $\tilde{\delta}, \tilde{\vartheta}$ be fuzzy sets defined as follows:

$$
\begin{array}{ccc}
\mu_{\widetilde{\delta}}(\alpha)=0.3 & \mu_{\widetilde{\delta}}(\beta)=0.4 & \mu_{\widetilde{\delta}}(\lambda)=0.5 \\
\mu_{\widetilde{\vartheta}}(\rho)=0.6 & \mu_{\widetilde{\vartheta}}(\sigma)=0.5 & \mu_{\widetilde{\vartheta}}(\omega)=0.2
\end{array}
$$

Let $\tilde{\tau}=\{\tilde{0}, \tilde{1}, \tilde{\delta}\}, \tilde{v}=\{\tilde{0}, \tilde{1}, \tilde{\vartheta}\}$ so, $(X, \tilde{\tau})$ and $(Y, \tilde{v})$ are f.t.s. Then the function $f:(X, \tilde{\tau}) \rightarrow$ $(Y, \tilde{v})$ defined by $f(\alpha)=\rho, f(\beta)=\sigma, f(\lambda)=\omega$ is f.s.p.co.f but not f.co.f. and f.s.p*..co.f.

\section{Remark 3.15:}

The following diagram explains the relationship among some fuzzy continuous function.

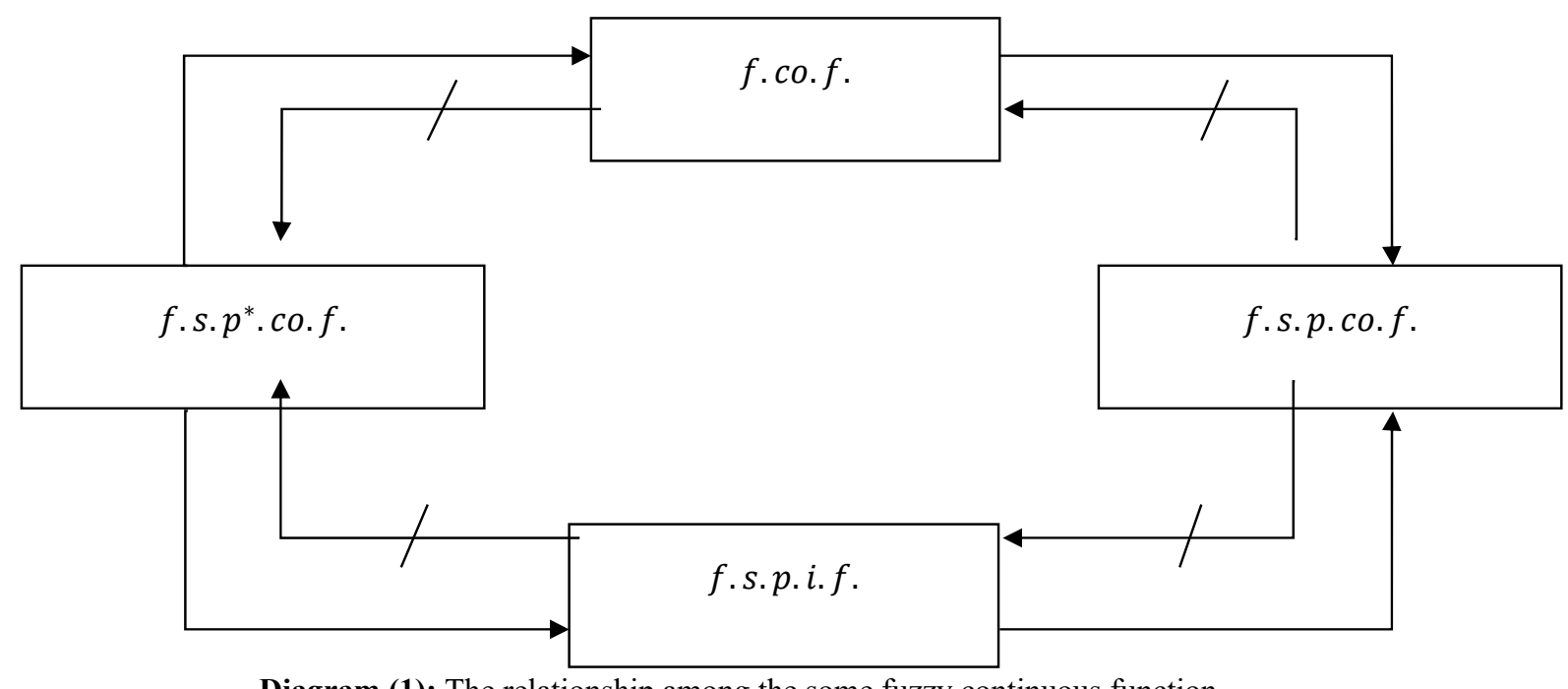

Diagram (1): The relationship among the some fuzzy continuous function 


\section{Fuzzy Semi Pre Homeomorphism in fuzzy Topological Spaces.}

In this section we introduce the fuzzy semi pre homeomorphism functions and fuzzy semi pre* homeomorphism functions in fuzzy topological spaces. Some of their properties and characterization have been proved and discussed in details.

\section{Definition 4.1:}

Let $f:(X, \tilde{\tau}) \longrightarrow(Y, \tilde{v})$ be bijection fuzzy function, $f$ is called a fuzzy semi pre homeomorphism iff $f$ and $f^{-1}$ are f.s.p.co.fs. (abbreviated as f.s.p.h.f.)

\section{Theorem 4.2:}

Every fuzzy homeomorphism function is a fuzzy semi pre homeomorphism function.

\section{Proof:}

Let $f:(X, \tilde{\tau}) \longrightarrow(Y, \tilde{v})$ be a fuzzy homeomorphism function. So, $f$ and $f^{-1}$ are fuzzy continuous functions. Hence, by Proposition (3.11) $f$ and $f^{-1}$ are fuzzy semi pre continuous functions. Thus, $f$ is a fuzzy semi pre homeomorphism

The converse of (Theorem (4.2)) is not true in general, so the below example explain that.

\section{Example 4.3:}

Let $X=\{x, y, z\}, Y=\{\alpha, \beta, \sigma\}$ and let $\tilde{A}, \tilde{B}$ be fuzzy sets defined as follows:

$$
\begin{array}{cll}
\mu_{\tilde{A}}(x)=0.2 & \mu_{\tilde{A}}(y)=0.3 & \mu_{\tilde{A}}(z)=0.5 \\
\mu_{\tilde{B}}(\alpha)=0.5 & \mu_{\tilde{B}}(\beta)=0.4 & \mu_{\tilde{B}}(\sigma)=0.1
\end{array}
$$

Let $\tilde{\tau}=\{\tilde{0}, \tilde{1}, \tilde{A}\}, \tilde{v}=\{\tilde{0}, \tilde{1}, \tilde{B}\}$ so, $(X, \tilde{\tau})$ and $(Y, \tilde{v})$ are f.t.s. Then the function $f:(X, \tilde{\tau}) \rightarrow$ $(Y, \tilde{v})$ defined by $f(x)=\alpha, f(y)=\beta, f(z)=\sigma$ is f.s.p.h. $f$ but not $f . h . f$. since $f$ and $f$ ${ }^{-1}$ are not $f$.co.f.

\section{Theorem 4.4:}

Let $f:(X, \tilde{\tau}) \longrightarrow(Y, \tilde{v})$ be bijection fuzzy function then the below statements are equivalent.

(1) $f$ is a fuzzy semi pre-open function.

(2) $f$ is a fuzzy semi pre closed function.

(3) $f^{-1}$ is a fuzzy semi pre continuous function.

\section{Proof:}

$(1) \rightarrow(2)$ Let $\tilde{\rho}$ be fuzzy closed set belong to $X$, then $\left(1-\mu_{\widetilde{\rho}}(x)\right)$ is a fuzzy open set belong to $\mathrm{X}$ since $f$ is a fuzzy semi pre-open function. Then $f\left(1-\mu_{\widetilde{\rho}}(x)\right) \in$ F.S.P.O. $(Y)$. So, by [Theorem (2.4)] we obtain $\left(1-\left(\mu_{f(\widetilde{\rho})}(f(x))\right)=\right.$ $\left(1-\left(\mu_{f(\widetilde{\rho})}(y)\right) \in\right.$ F.S.P.O. $(Y)$. Thus, by [Theorem (3.3)] $\left(\mu_{f(\widetilde{\rho})}(y)\right) \in$ F.S.P.C. $(Y)$. Hence, $f$ is a fuzzy semi pre closed function

$(2) \rightarrow(3)$ Let $\tilde{\delta}$ be any fuzzy open set belong to X. So, $\left(1-\mu_{\widetilde{\delta}}(x)\right)$ is a fuzzy closed set belong to $\mathrm{X}$. but $f$ is a fuzzy semi pre closed function. So, $f\left(1-\mu_{\widetilde{\delta}}(x)\right) \in$ F.S.P.C. $(Y)$ By [Theorem (2.4)] we obtain $\left(1-\left(\mu_{f(\widetilde{\delta})}(f(x))\right)=\left(1-\left(\mu_{f(\widetilde{\delta})}(y)\right) \in\right.\right.$ F.S.P.C. $(Y)$. Thus, by [Theorem (3.3)] $\left(\mu_{f(\widetilde{\delta})}(y)\right)=\mu_{\left(f^{-1}\right)^{-1}(\widetilde{\delta})}(y) \in$ F.S.P.O. $(Y)$. Hence, $f^{-1}$ is a fuzzy semi pre continuous function 
$(3) \rightarrow(1)$ Let $\widetilde{\omega}$ be any fuzzy open set belong to $X$. and since, $f^{-1}$ is a fuzzy semi pre continuous function. So, $\mu_{\left(f^{-1}\right)^{-1}(\widetilde{\omega})}(y)=\left(\mu_{f(\widetilde{\omega})}(y)\right) \in$ F.S.P.O. $(Y)$. Hence, $f$ is a fuzzy semi pre-open function

\section{Corollary 4.5:}

Let $f:(X, \tilde{\tau}) \longrightarrow(Y, \tilde{v})$ be a bijective fuzzy function, then the below statements are equivalent.

(1) $f$ is a fuzzy semi pre continuous and a fuzzy semi pre-open functions.

(2) $f$ is a fuzzy semi pre continuous and a fuzzy semi pre closed functions.

(3) $f$ is a fuzzy semi pre homeomorphism function.

\section{Proof:}

$(1) \rightarrow$ (2) Follows from proof Theorem (4.4) [(1) $\rightarrow$ (2)]

(2) $\rightarrow$ (3) Follows from proof Theorem (4.4) [(2) $\rightarrow$ (3)]

(3) $\rightarrow$ (1) Follows from Definition (4.1) and proof Theorem (4.4) [(3) $\rightarrow(1)]$

\section{Definition 4.6:}

Let $f:(X, \tilde{\tau}) \longrightarrow(Y, \tilde{v})$ be bijection fuzzy function, $f$ is called a fuzzy semi pre* homeomorphism iff $f$ and $f^{-1}$ are fuzzy semi pre irresolute functions (abbreviated as $f . s . p^{*} . h . f$. ).We say the spaces $(X, \tilde{\tau})$ and $(Y, \tilde{v})$ are fuzzy semi pre* homeomorphism if there exist a fuzzy semi pre* homeomorphism from $(X, \tilde{\tau})$ on to $(Y, \tilde{v})$.

The family of fuzzy semi pre* homeomorphism from a f.t.s $(X, \tilde{\tau})$ to itself is denoted by fuzzy semi pre* Homeomorphism $(X, \tilde{\tau})$ (abbreviated as f.s. $\left.p^{*} . h .(X, \tilde{\tau})\right)$.

\section{Theorem 4.7}

Every fuzzy semi pre* homeomorphism function is a fuzzy semi pre homeomorphism function.

\section{Proof:}

Let $f:(X, \tilde{\tau}) \longrightarrow(Y, \tilde{v})$ be a fuzzy semi pre* homeomorphism function, So, $f$ and $f^{-1}$ are fuzzy semi pre irresolute functions. Hence, by Proposition (3.11) $f$ and $f^{-1}$ are fuzzy semi pre continuous functions. Thus, $f$ is a fuzzy semi pre homeomorphism function

\section{Theorem 4.8:}

Let $f:(X, \tilde{\tau}) \longrightarrow(Y, \tilde{v})$ be a bijective fuzzy function, then the below statements are equivalent.

(1) $f$ is a fuzzy semi pre irresolute and a fuzzy semi pre*- open functions.

(2) $f$ is a fuzzy semi pre irresolute and a fuzzy semi pre* closed functions.

(3) $f$ is a fuzzy semi pre* homeomorphism function.

\section{Proof:}

$(1) \rightarrow(2)$ Let $\tilde{\rho} \in$ F.S.P.C. $(X)$.Then $\left(1-\mu_{\tilde{\rho}}(x)\right) \in$ F.S.P.O. $(X)$ since $f$ is a fuzzy semi pre*- open function. Then $f\left(1-\mu_{\widetilde{\rho}}(x)\right) \in$ F.S.P.O.(Y). So, by [Theorem (2.4)] we obtain $\left(1-\left(\mu_{f(\widetilde{\rho})}(f(x))\right) \quad=\left(1-\left(\mu_{f(\widetilde{\rho})}(y)\right) \in\right.\right.$ F.S.P.O. $(Y)$.Thus, $\left.\mu_{f(\widetilde{\rho})}(y)\right) \in$ 
F.S.P.C. $(Y)$. Hence, $f$ is a fuzzy semi pre irresolute and a fuzzy semi pre* closed functions

$(2) \rightarrow(3)$ Let $\tilde{\delta} \in$ F.S.P.O. (X). So, $\left(1-\mu_{\widetilde{\delta}}(x)\right) \in$ F.S.P.C. $(X)$. but $f$ is a fuzzy semi pre* closed functions. So, $f\left(1-\mu_{\widetilde{\delta}}(x)\right) \in$ F.S.P.C. $(Y)$. By [Theorem (2.4)] we obtain $\left(1-\left(\mu_{f(\widetilde{\delta})}(f(x))\right)=\left(1-\left(\mu_{f(\widetilde{\delta})}(y)\right) \in F . S . P . C .(Y)\right.\right.$.Thus, $\left(\mu_{f(\widetilde{\delta})}(y)\right)=\mu_{\left(f^{-1}\right)^{-1}(\widetilde{\delta})}(y) \in$ F.S.P.O. $(Y)$. Hence, $f^{-1}$ is a fuzzy semi pre irresolute function and we know $f$ is a fuzzy semi pre irresolute function. Therefor; $f$ is a fuzzy semi pre* homeomorphism function $(3) \rightarrow$ (1) Let $\widetilde{\omega} \in$ F.S.P.O.(X).Since, $f^{-1}$ is a fuzzy semi pre irresolute function So, $\mu_{\left(f^{-1}\right)^{-1}(\widetilde{\omega})}(y)=\left(\mu_{f(\widetilde{\omega})}(y)\right) \in$ F.S.P.O. $(Y)$. Hence, by [definition (4.6)] $f$ is a fuzzy semi pre irresolute function and a fuzzy semi pre*-open function

\section{Theorem 4.9:}

Let $f:(X, \tilde{\tau}) \longrightarrow(Y, \tilde{v})$ and $h:(Y, \tilde{v}) \longrightarrow(Z, \tilde{u})$ are two fuzzy semi pre* homeomorphism function then the function $h \circ f:(X, \tilde{\tau}) \longrightarrow(Z, \tilde{u})$ is also a fuzzy semi pre* homeomorphism function.

\section{Proof:}

Let $\mu_{\widetilde{\alpha}}(z) \leq \mu_{z}(z) \in$ F.S.P.O. $(Z)$, since $h$ is a fuzzy semi pre irresolute function. So, $\mu_{h^{-1}(\widetilde{\alpha})}(y) \in$ F.S.P.O. $(Y)$ also since $f$ is a fuzzy semi pre irresolute function. So, we obtain $\mu_{f^{-1}\left(h^{-1}(\widetilde{\alpha})\right)}(x)=\mu_{(h \circ f)^{-1}(\widetilde{\alpha})}(x) \in F . S . P . O .(X)$. Thus, $h \circ f$ is a fuzzy semi pre irresolute function.

And let $\mu_{\widetilde{\beta}}(x) \leq \mu_{x}(x) \in F . S . P . O .(X)$, since $f^{-1}$ is a fuzzy semi pre irresolute function. So, $\mu_{\left(f^{-1}\right)^{-1}(\widetilde{\beta})}(y)=\mu_{f(\widetilde{\beta})}(y) \in$ F.S.P.O. $(Y)$ and since $h^{-1}$ is a fuzzy semi pre irresolute function,So, $\mu_{\left(h^{-1}\right)^{-1}(f(\widetilde{\beta}))}(z)=\mu_{h(f(\widetilde{\beta}))}(z)=\mu_{(h \circ f)(\widetilde{\beta})}(z)=\mu_{\left((h \circ f)^{-1}\right)^{-1}(\widetilde{\beta})}(z) \in$ F.S.P.O.(Z). Thus, $(h \circ f)^{-1}$ is a fuzzy semi pre irresolute function. Therefore, $h \circ f$ is a fuzzy semi pre* homeomorphism function

\section{Theorem 4.10:}

Let $(X, \tilde{\tau})$ be the set of all fuzzy semi pre* homeomorphism (or $f . s . p^{*} . h .(X, \tilde{\tau})$ for short), then $(X, \tilde{\tau})$ is a group under the usual composition functions.

\section{Proof:}

Assume that $*: f . s . p^{*} . h .(X, \tilde{\tau}) \times f . s . p^{*} . h .(X, \tilde{\tau}) \rightarrow f . s . p^{*} . h .(X, \tilde{\tau})$ is a binary operation function defined by $\mu_{g * f}(x)=\mu_{g \circ f}(x)$, for all $\mu_{f}(x), \mu_{g}(x) \leq \mu_{f . s . p^{*} . h .(X, \tilde{\tau})}(x)$. Now by the above [Theorem (4.9)], we get $\mu_{g \circ f}(x) \leq \mu_{f . s . p^{*} . h .(X, \tilde{\tau})}(x)$ i.e, $(*)$ is closed and by usual definition of composition functions we know $(*)$ is associative and there exists the identity function I:f.s. $p^{*} . h .(X, \tilde{\tau}) \rightarrow f . s . p^{*} . h .(X, \tilde{\tau})$ such that $\mu_{f \circ I}(x)=\mu_{I \circ f}(x)=\mu_{f}(x)$ i.e., $\mu_{f * I}(x)=\mu_{I * f}(x)=\mu_{f}(x)$ and finely for all $\mu_{f}(x) \leq \mu_{f . s . p^{*} . h .(X, \tilde{\tau})}(x)$ there exists $\mu_{f^{-1}}(x) \leq$ $\mu_{f . s . p^{*} . h .(X, \tilde{\tau})}(x)$ such that $\mu_{f \circ f^{-1}}(x)=\mu_{f^{-1} \circ f}(x)=\mu_{I}(x)$ i.e, $\mu_{f * f^{-1}}(x)=\mu_{f^{-1} * f}(x)=$ $\mu_{I}(x)$.Thus it's clear $\left(f . s . p^{*} . h .(X, \tilde{\tau}), \circ\right)$ is a group 


\section{Conclusions}

In this paper, we been developed the new types of fuzzy homeomorphism function in fuzzy topological spaces called fuzzy semi pre homeomorphism function and fuzzy semi pre* homeomorphism function. In addition to that we developed the relationship between these new types of fuzzy homeomorphism function in fuzzy topological spaces with some new kinds of fuzzy semi pre continuous function. Finally, we structure a group under the usual composition functions by using these new types of fuzzy homeomorphism function. As follows:

(I) Every fuzzy semi pre* homeomorphism function is a fuzzy semi pre homeomorphism function.

(II) Let $f:(X, \tilde{\tau}) \longrightarrow(Y, \tilde{v})$ be a bijective fuzzy function, then the below statements are equivalent.

(1) $f$ is a fuzzy semi pre irresolute and a fuzzy semi pre*-open functions.

(2) $f$ is a fuzzy semi pre irresolute and a fuzzy semi pre* closed functions.

(3) $f$ is a fuzzy semi pre* homeomorphism function.

(III) Let $(X, \tilde{\tau})$ be the set of all fuzzy semi pre* homeomorphism (or f.s.p*.h. $(X, \tilde{\tau})$ for short), then $(X, \tilde{\tau})$ is a group under the usual composition functions.

\section{References}

1. Zadeh, L.A. Fuzzy Sets. Inform. Control. 1965, 8, 338-353.

2. Chang, C. L. Fuzzy Topological Spaces. J. Math. Anal. Appl. 1968, 24,182- 190.

3. Ming, P. P. ; Ming, L. Y. Fuzzy Topology I. Neighborhood Structure of a Fuzzy point and Moor-smith Convergence. J. Math. Anal. Appl. 1980, 76,571-599.

4. Andrijevic, D. Semi preopen sets. Mat.Vesnik.1986, 38, 24- 32.

5. Thakur, S. S. ; Singh, S. On Fuzzy Semi-Preopen Sets and Fuzzy Semi-Precontinuity. J. Fuzzy Sets and Systems. 1998, 98, 383-391.

6. Mageed, S. Y. On Fuzzy Compact Spaces. M.Sc. Thesis, College of Education, AlMustansiryah University, 2012.

7. Ali, T.; Das, S. Fuzzy Topological Transformation Groups. J. Math. Rese.2009,1, $1,78-86$.

8. Cahit, T. Some Properties of Fuzzy Contınuity Functions. African Journal of Mathematics and Computer Science Research. 2017, 10,2, 14-17, doi: 10.5897/AJMCSR2017.00691.

9. Satyamurthy, V. P.; Sadanand, N. P. On Some forms of fuzzy wg**- Continuous functions in Fuzzy Topological Spaces. Int. J. Adv. Sci. Eng. 2018, 5, 2, 901-905, doi:10.29294/IJASE.5.2.2018.901-905.

10. Balasubramaniyan, K.; Sriram, S.; Ravi ,O .On Fuzzy Almost Contra $\gamma$-Continuous Functions. International Journal of Pure and Applied Mathematics. 2013, 85, 1, 109127, doi:10.12732/ijpam.v85i1.9.

11. Thiruchelvi, M. ; Ilango, G. Fuzzy Generalized Semi Preregular Continuous Functions in Fuzzy Topological Spaces. International Journal of Pure and Applied Mathematics. 2016, 106, 6, 75-83. 